\title{
Prevalence of preoperative anxiety among Hausa patients undergoing elective surgery-a descriptive study
}

\author{
Sabo Saleh Dagona \\ Yobe State University, \\ PMB 1144, Damaturu, Yobe State, Nigeria.
}

\begin{abstract}
The aim of this study is to assess the prevalence of preoperative anxiety among Nigerian Hausa patients undergoing elective surgery. Methods: Thirty patients between the ages of 17- 64 years scheduled to undergo elective surgery in a tertiary health facility in northern Nigeria were recruited and administered a translated Hausa version of the Amsterdam Preoperative Anxiety and Information Scale (APAISH).APAIS-H was administered to each patient in the evening before and in the morning of their surgery day. Results: Statistical Package for Social Sciences (SPSS) Version 23.0 was used to analyse the data. Analyses were according to the APAIS subscales to see preoperative anxiety a day before and on the day of surgery. On both the fear of anaesthesia and fear of surgery subscales, $76.6 \%$ of the participants presented with low to moderate anxiety in the evening proceeding the day of their surgery. Only $23.3 \%$ of the participants were highly anxious of either anaesthesia or surgery in the evening proceeding the day of their surgery. On the anxiety related to surgery subscale, in the morning of the surgery day, $36.7 \%$ of the participants had low to moderate anxiety related to anaesthesia while $63.3 \%$ were highly anxious about anaesthesia. Also, $40 \%$ of the participants presented with low to moderate anxiety related to surgery in the morning of their surgery while the remaining $60 \%$ were highly anxious of the surgery in the morning of the day of their surgery. The full scale preoperative anxiety scores reveal that, $53 \%$ of the participants had low anxiety, 26.7 had moderate anxiety while $20 \%$ participants had high preoperative anxiety in the evening preceding their surgery. In the morning of the day of their scheduled surgery, $20 \%$ of the participants presented with low anxiety, another $20 \%$ had moderate anxiety while the remaining $60 \%$ had high preoperative anxiety. Conclusion: The findings of the study show that, anxiety before elective surgery is prevalent among the Hausa patients. It is therefore recommended that, adequate psychological preoperative anxiety reduction interventions be put in place to address patients' anxiety to achieve a better surgical outcome.
\end{abstract}

\section{INTRODUCTION}

Anxiety at the preoperative period is a major problem among surgical patients. Preoperative anxiety is described as an incapacitating state of uneasiness, tension, apprehension, dread and nervousness disproportionate to the threat of surgery being confronted (Pritchard 2009a; Ali et al. 2014). The prevalence rate of preoperative anxiety differs among surgical patients and based on the type of surgery, previous surgical experience and the patients' coping mechanisms. Studies have shown that the prevalence of preoperative anxiety is estimated at between 60\% -80\% among western surgical patients (Matthias and Samarasekera 2012). Gangadharan et al. (2014) administered State Trait Anxiety Inventory (STAI) to evaluate preoperative anxiety in Saudi Arabian patients before elective surgery. The results revealed that $60 \%$ of the patients reported high anxiety; $30 \%$ moderate anxiety and $10 \%$ low anxiety prior to surgery. Akinsulore et al. (2015) assessed pre-and post-operative anxiety among fifty one elective major surgery patients in a tertiary hospital in Nigeria. The findings revealed that $51.0 \%$ of the patients had significantly high pre-operative anxiety, whereas $15.7 \%$ presented 
with significantly high post-operative anxiety. In another study, (Jafar and Khan 2009) studied the frequency of preoperative anxiety in Pakistani surgical patients. The aim of the study was to measure how frequent preoperative anxiety is among surgical patients and to correlate the visual analogue scale (VAS) and the state trait anxiety inventory (STAI). The study results revealed that $62 \%$ of the total participants were significantly anxious, preoperatively.

\section{Causes of preoperative anxiety}

Preoperative anxiety remains a source of acute stress among patients undergoing surgical operation. Preoperative anxiety disrupts the patients' attention and concentration and affects their overall experience of the surgery. A number of factors have been reported as being responsible for preoperative anxiety among surgical patients. Negative expectations of what may happen intra-operatively or postoperatively, and the fear of pain, body mutilation, loss of control and freedom, are documented as some of the causes of anxiety among surgical patients (Bailey 2010; Aziato and Adejumo 2014). Other causes of preoperative anxiety are signs of causal diseases such as diabetes and hypertension, information from family members and friends, and past experience of anaesthesia and surgical operations (Wakim et al. 2010). Perks et al. (2009) found that common causes of preoperative anxiety in surgical patients include waiting for the surgery, expectation of postoperative pain, fear of the operation and of postsurgical incapacitation. Other studies also reported that general anaesthesia, the induction of anaesthesia and being under the anaesthetic mask and fear of needles during the induction of anaesthesia are the most reported source of patients' preoperative anxiety (Ward et al. 2007; Jakobsson et al. 2008). In an interview conducted an interview with 240 patients scheduled to undergo day surgery van den Berg et al. (2005), found that, adult day surgical patients are more comfortable with inhalation induced anaesthesia to intravenous induction. Mitchell (2009) also reported that many surgical patients who go through regional and local anaesthesia were anxious of awaking up in theatre.

\section{Effects of preoperative anxiety}

The effects of preoperative anxiety have been reported in the literature with some studies showing both psychological and physical consequences on the patients. The psychological effects of preoperative anxiety include irritability, hostility, unfriendliness and being highly demanding and they develop more attention-seeking behaviours. The physical effects of preoperative anxiety include physiological changes which affect the functions of the central nervous system and the immune system (G. Chakir 2017). It has been reported that preoperative anxiety increases the release of catecholamine, resulting in physical changes such as increased heart rate, narrowed blood vessels, hyperventilation and blood setting with fast coagulation. Catecholamine is released to prepare the body for 'flight or fight' in response to a physical threat. A higher release of catecholamine causes medical problems like muscle tremors, diabetes, heart attack and stroke. Higher catecholamine in the body decreases the functions of the immune system (Wetsch et al. 2009), causing susceptibility to opportunistic infections and prolonged surgical wound healing. In some patients the physical manifestation of preoperative anxiety is in the form of dizziness or tiredness, irregular respiration and exaggerated feelings of pain (Bilberg et al. 2012). Other patients may feel wobbly or shaky, present with headache, stomach upsets or diarrhoea, nausea and vomiting and difficulty in the induction of and poor responses to anaesthesia (NHS 2015). Such symptoms can lead to despondent feeling, lack of energy and motivation which if not controlled can progress to a feeling of depression (Willson and Veale 2009: P-9). Bilberg et al. (2012) explained that balance functioning and emotions have similar neurological pathways. Therefore, lengthy dizziness and imbalance in most surgical patients are caused by preoperative anxiety. Because preoperative anxiety affect the functions of the immune system, it causes susceptibility to opportunistic postoperative infections, inflammation of the surgical site, leading to the 
consumption of higher doses of antibiotics and analgesics (Pritchard 2009b; May 2014). The cumulative effects amount to delaying the discharge of patients from the hospital and increasing their cost of medication. This study was designed to find out the prevalence of preoperative anxiety among Hausa speaking patients undergoing elective surgery

\section{Participants}

\section{METHOD}

Thirty participants, comprising of 17 male and 13 female were recruited to participate in the study. Their ages ranged from 17 to 70 years (mean age $=41.03$ and standard deviation $=$ 16.09). Of the total number of participants, 8 are single, 19 married, and the remaining 3 participants were either divorced or separated. Fourteen participants are unemployed, 15 employed and one is retired. The educational backgrounds of the participants varied, with one student, 2 participants had primary education, 6 had secondary and 9 have education up to tertiary level. On the other hand, 12 participants had informal education (either adult education or Qur'anic education)

\section{Recruitment of participants}

In the hospital setting where the study was conducted, elective surgeries are performed twice a week - on Tuesdays and Thursdays. Patients on the waiting list arrive at the hospital on Mondays or Wednesdays to receive instructions about their operations and undergo other necessary preoperative preparations. To recruit patients for the study, the researcher was based in the consulting clinic where patients on the waiting list come to see the surgeons or nurses before the day of their scheduled surgical operations. After consulting with their doctors, surgeons or nurses, the researcher approached the patients and informed them about the research and sought their participation. Patients who agreed to participate were enrolled. Consent forms and information sheets containing details about the research and what was required as they participate were also given to them to read and sign. The participants were reminded about the right to discontinue participation in the study at any time and that this would not affect the service they received in the hospital. Through this process, a total of 30 participants were recruited to participate in the study.

\section{Instrument}

The instrument used in this study was the translated Hausa version of the Amsterdam Preoperative Anxiety and Information Scale (APAIS-H). APAIS was developed by (Moerman et al. 1996) to serve as a short screening tool to be used by clinicians in assessing the preoperative anxiety of patients undergoing surgical operation (Moerman et al. 1996). The instrument has been used in different clinical settings and for different research purposes. APAIS has been found to be a valid and very reliable means of measuring preoperative anxiety. This scale was translated into the Hausa language by the researcher for the purpose of this study. The translation was carried out by two independent professional translators using the forward-backward translation method, synthesis of the forward translation and expert committee review before arriving at the final translated Hausa version. The translated Hausa version was then pilot tested on real patients scheduled for elective surgery. After pilot study the instrument was used to collect again on patients scheduled to undergo surgical operation. The data collected was used to calculate the validity and reliability indexes of the translated Hausa version of the scale (APAIS-H). Psychometric evaluation found the scale to be highly reliable, with Cronbach's alpha 0.82 , which was similar to that of the original scale developed by (Moerman et al. 1996). The study participants were also questioned through a structured interview to indicate their level of understanding of the translated Hausa version of the questionnaire. The responses obtained for each item indicated that it was either understood or very well understood. 


\section{Study setting and ethical clearance.}

The study was conducted in a tertiary hospital in North-eastern Nigeria. The hospital performs elective surgeries on patients with different medical and surgical conditions. Ethical approval for this study was granted by the ethics committee of the Hospital following the application for ethical clearance filed by the researchers to obtain the formal permission to conduct this research.

\section{Data collection}

\section{PROCEDURE}

Data collection occurred in the hospital ward environment, which was reasonably free from any form of distractions. For each of the participants, data was collected during two time periods: during the evening before the scheduled surgery and on the morning of the day of their surgery. In either case, each of the participants was given the questionnaire APAIS-H to read and score by himself/herself. Those participants who could not read and understand the questionnaire by themselves had it read to them by the researcher and their responses were recorded. After responding to the questionnaire during the evening before the day of their surgeries, participants were also interviewed by the researcher to find out the specific things they feared most about their planned surgery and why.

\section{RESULTS}

A descriptive statistics using frequency counts and percentage was used to analyse the data obtained from the study participants in the evening before and in the morning of the day of surgery. The data is presented in tabular and graphical forms in accordance with the subscales of the instrument, APAIS-H. For each subscale, the participants' scores were categorised as low, moderate or high. Finally, full-scale preoperative anxiety scores for the participants was presented also tabular and graphical form. Accordingly, the participants overall preoperative anxiety was categorised as either low, moderate or high based on what was obtained from the participants APAI-H scores.

\section{Anxiety related to anaesthesia}

In the evening before and on the morning of their scheduled surgery, it can be seen most of the participants' fear of anaesthesia were between low and moderate levels. A total of 13 (43.3\%) participants presented with low anaesthesia related anxiety, 10 (33.3\%) had moderate anxiety levels while the remaining 7 (23.3\%) appeared to have high anxiety related to anaesthesia. On the other hand, on the morning of the patients' scheduled surgery, the data collected reveals, only $5(16.7 \%)$ participants presented with low levels of anxiety related to anaesthesia, 6 $(20.0 \%)$ had moderate anxiety, while a significant number-19, $(63.3 \%)$ of the participants had higher level of anxiety related to anaesthesia. Table 1 presents data on the participants' levels of anxiety related to the fear of anaesthesia

Table 3: Anxiety related to anaesthesia

\begin{tabular}{|cccccc|}
\hline \multicolumn{2}{|c|}{ Evening Before Surgery Day } & \multicolumn{3}{c|}{ Morning of Surgery Day } \\
\hline Levels of Anxiety & $\begin{array}{c}\text { Frequency } \\
(\mathbf{N})\end{array}$ & $\begin{array}{c}\text { Per cent } \\
(\mathbf{\%})\end{array}$ & Levels of Anxiety & $\begin{array}{c}\text { Frequency } \\
\text { (N) }\end{array}$ & $\begin{array}{c}\text { Per cent } \\
\text { (\%) }\end{array}$ \\
\hline Low & 13 & 43.3 & Low & 5 & 16.7 \\
\hline Moderate & 10 & 33.3 & Moderate & 6 & 20.0 \\
\hline High & 7 & 23.3 & High & 19 & 63.3 \\
\hline Total & $\mathbf{3 0}$ & $\mathbf{1 0 0}$ & Total & $\mathbf{3 0 .}$ & $\mathbf{1 0 0}$ \\
\hline
\end{tabular}

The data for anxiety related to anaesthesia is summarised and presented in the bar chart below. 


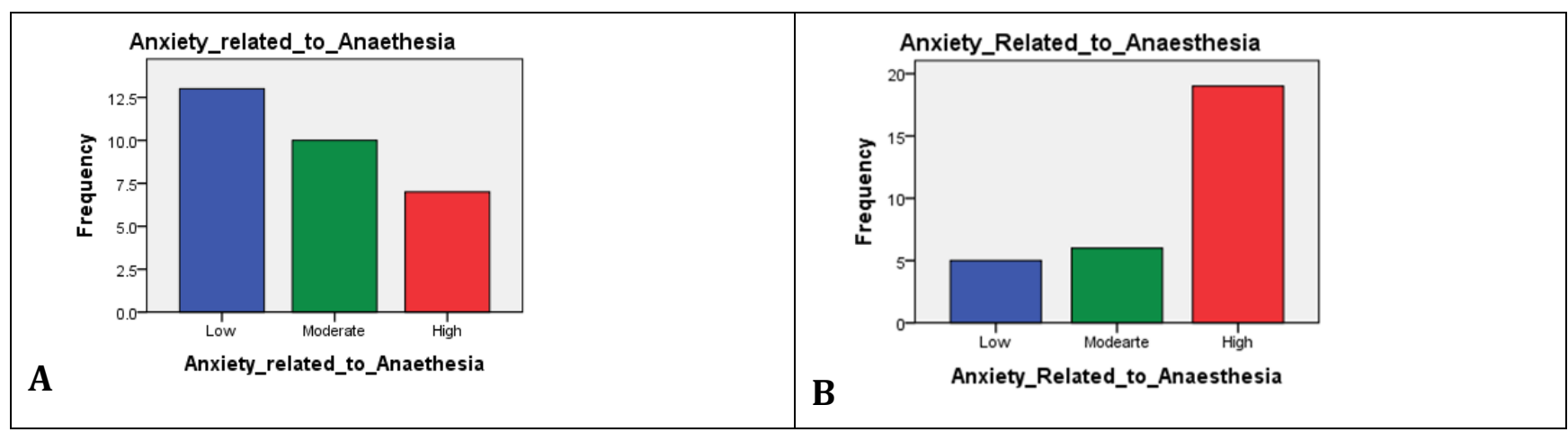

Figure 10: Anxiety related to anaesthesia during the evening before surgery day. $B=$ Anxiety related to surgery on the morning of the surgery day.

\section{Anxiety related to surgery}

The participants' levels of anxiety related to surgical operation on the evening before their surgery shows that 10 (33.3\%) participants had low anxiety related to surgery; 13 participants $(43.3 \%)$ presented with moderate anxiety related to surgery, while 7 participants (23.3\%) reported high anxiety related to surgery. Conversely, in the morning of the day of their surgery, participants' anxiety related to surgery subscale show a remarkable difference in. Of the 30 participants, only $4(13.3 \%)$ reported having low anxiety related to surgery; 8 participants (26.7\%) presented with moderate surgical anxiety and a greater proportion 18, (60\%) recorded having high anxiety related to surgery. In summary, a total of 23 participants reported either low or moderate anxiety related to surgery in the evening before their surgery or only 7 participants presented with high anxiety. On the other hand only $12,(40 \%)$ out of the participants presented with low to moderate anxiety related to surgery in the morning of their scheduled surgery, while 18 (60\%) participants presented with high surgical anxiety on the morning of the day of their surgical operation. Table 2 presents data on the participants' levels of anxiety related to surgical operation obtained in the evening before and in the morning of the day of surgery.

Table 4: anxiety related to surgery

\begin{tabular}{|cccccc|}
\hline Evening Before Surgery Day & \multicolumn{5}{c|}{ Morning of Surgery Day } \\
$\begin{array}{c}\text { Levels of } \\
\text { Anxiety }\end{array}$ & $\begin{array}{c}\text { Frequency } \\
(\mathbf{N})\end{array}$ & $\begin{array}{c}\text { Per cent } \\
\text { (\%) }\end{array}$ & $\begin{array}{c}\text { Levels of } \\
\text { Anxiety }\end{array}$ & $\begin{array}{c}\text { Frequency } \\
\text { (N) }\end{array}$ & $\begin{array}{c}\text { Per cent } \\
\text { (\%) }\end{array}$ \\
\hline Low & 10 & 33.3 & Low & 4 & 13.3 \\
\hline Moderate & 13 & 43.3 & Moderate & 8 & 26.7 \\
\hline High & 7 & 23.3 & High & 18 & 60.0 \\
\hline Total & $\mathbf{3 0}$ & $\mathbf{1 0 0}$ & Total & $\mathbf{3 0}$ & $\mathbf{1 0 0}$ \\
\hline
\end{tabular}

Figure 2 summarises the data presented in the table 2 above.

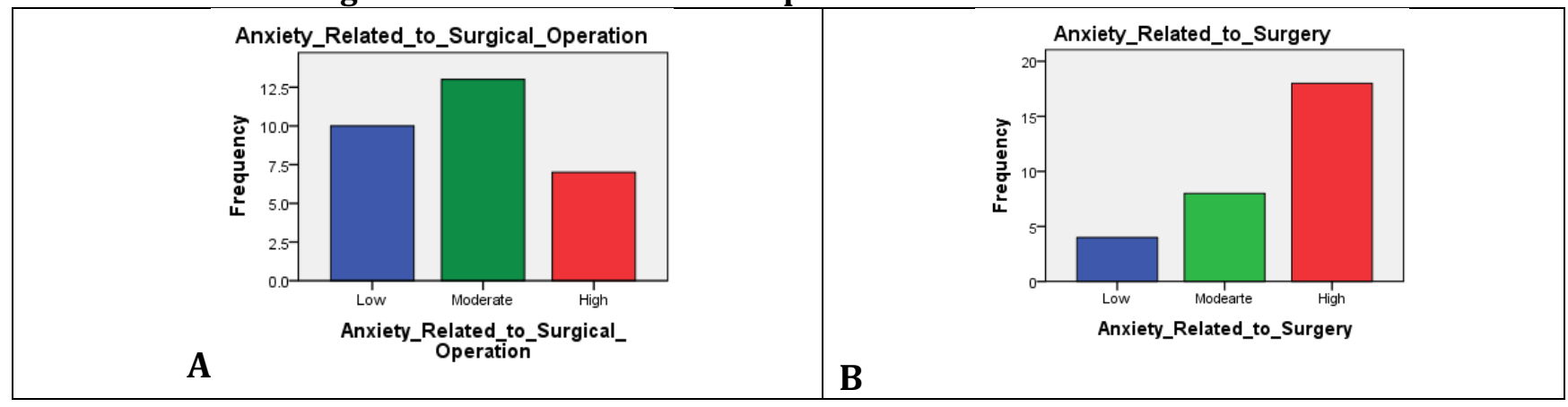

Figure 2: $A=$ Anxiety related to surgery during the evening before the surgery day. $B=$ Anxiety related to surgery on the morning of the surgery day 
On the information desire sub-scale, the data collected revealed that in the evening before the day of surgery, only $5(16.7 \%)$ participants presented with low desire for information about their surgery, 8 (26.7\%) participants had moderate desire for surgical information, and more than half of participants 17 (56.7\%) reported a higher need for information about their surgery. On the other hand, on the morning of the day of their surgery, no patient reported having low desire for information about surgery. Patients reported between moderate and higher need for information, with only 7 (23.3\%) participants presenting with moderate need for information, while the remaining 27 participants (76.7\%) reported higher desire for surgical information. The data for information desire for surgical information is presented in table 3 below.

Table 3: Information desire about surgery

\begin{tabular}{|cccccc|}
\hline $\begin{array}{c}\text { Levels of } \\
\text { Info. } \\
\text { Desire }\end{array}$ & $\begin{array}{c}\text { Frequency } \\
\text { (N) }\end{array}$ & $\begin{array}{c}\text { Per cent } \\
\mathbf{( \% )}\end{array}$ & $\begin{array}{c}\text { Levels of } \\
\text { Info. } \\
\text { Desire }\end{array}$ & $\begin{array}{c}\text { Frequency } \\
\text { (N) }\end{array}$ & $\begin{array}{c}\text { Per cent } \\
\text { (\%) }\end{array}$ \\
\hline Low & 5 & 16.7 & Low & - & \\
\hline Moderate & 8 & 26.7 & Moderate & 7 & 23.3 \\
\hline High & 17 & 56.7 & High & 23 & 76.7 \\
\hline Total & $\mathbf{3 0}$ & $\mathbf{1 0 0}$ & Total & $\mathbf{3 0}$ & $\mathbf{1 0 0}$ \\
\hline
\end{tabular}

The data for information desire for surgical information is presented in the following graphs.

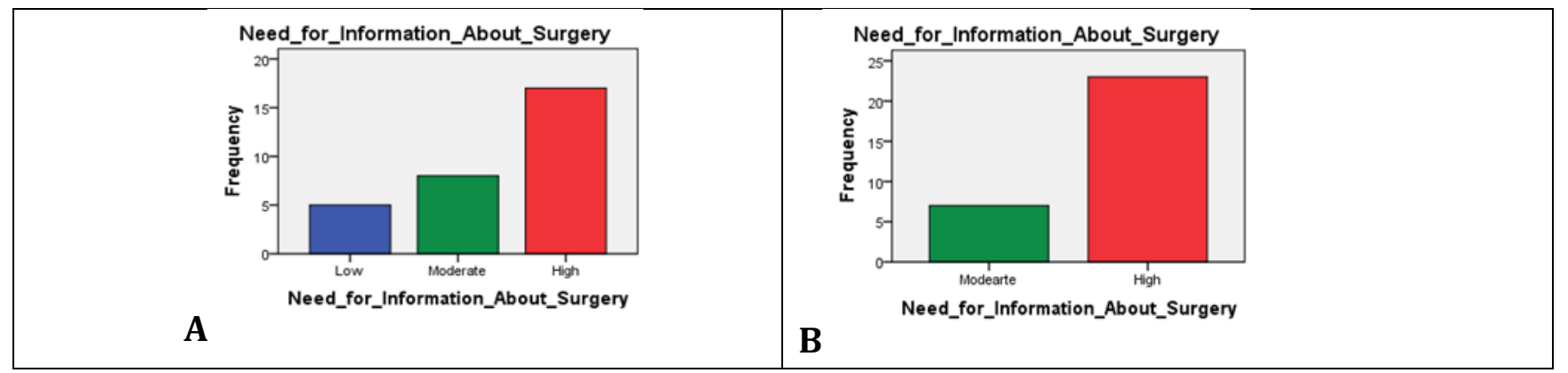

Figure 3: A= Participants' scores on the information desire sub-scale during the evening before the surgery day. B= Participants' scores on the information desire sub-scale on the morning of surgery day.

\section{Full-scale preoperative anxiety}

The full scale total anxiety scores of the study participants during the evening preceding their scheduled surgery shows that, 16 participants (53.3\%) presented with low levels of preoperative anxiety, $8(26.7 \%)$ had moderate anxiety and $6(20 \%)$ of the participants recorded high level of preoperative anxiety. On the morning of day of their surgical operation, participants' measured levels of preoperative anxiety reveal that only 6 participants (20\%) presented with low levels of preoperative anxiety, another $6(20 \%)$ presented with moderate levels of preoperative anxiety, and a total of eighteen participants $(18-60 \%)$ presented with high levels of preoperative anxiety. The information on participants' full scale preoperative anxiety is presented in the table below. 
Table 4: Participants' cumulative preoperative anxiety in the evening before and the on the morning of their surgery day

\begin{tabular}{|cccccc|}
\hline $\begin{array}{c}\text { Evening Before Surgery Day } \\
\begin{array}{c}\text { Preoperative } \\
\text { Anxiety }\end{array}\end{array}$ & $\begin{array}{c}\text { Frequency } \\
\text { (N) }\end{array}$ & $\begin{array}{c}\text { Per cent } \\
\text { (\%) }\end{array}$ & $\begin{array}{c}\text { Preoperative } \\
\text { Anxiety }\end{array}$ & $\begin{array}{c}\text { Frequency } \\
\text { (N) }\end{array}$ & $\begin{array}{c}\text { Per cent } \\
\text { (\%) }\end{array}$ \\
\hline Low & 16 & 53.3 & Low & 6 & 20.0 \\
\hline Moderate & 8 & 26.7 & Moderate & 6 & 20.0 \\
\hline High & 6 & 20.0 & High & 18 & 60.0 \\
\hline Total & $\mathbf{3 0}$ & $\mathbf{1 0 0 . 0}$ & Total & $\mathbf{3 0}$ & $\mathbf{1 0 0 . 0}$ \\
\hline
\end{tabular}

The information on participants' full scale preoperative anxiety is presented in the following chart.

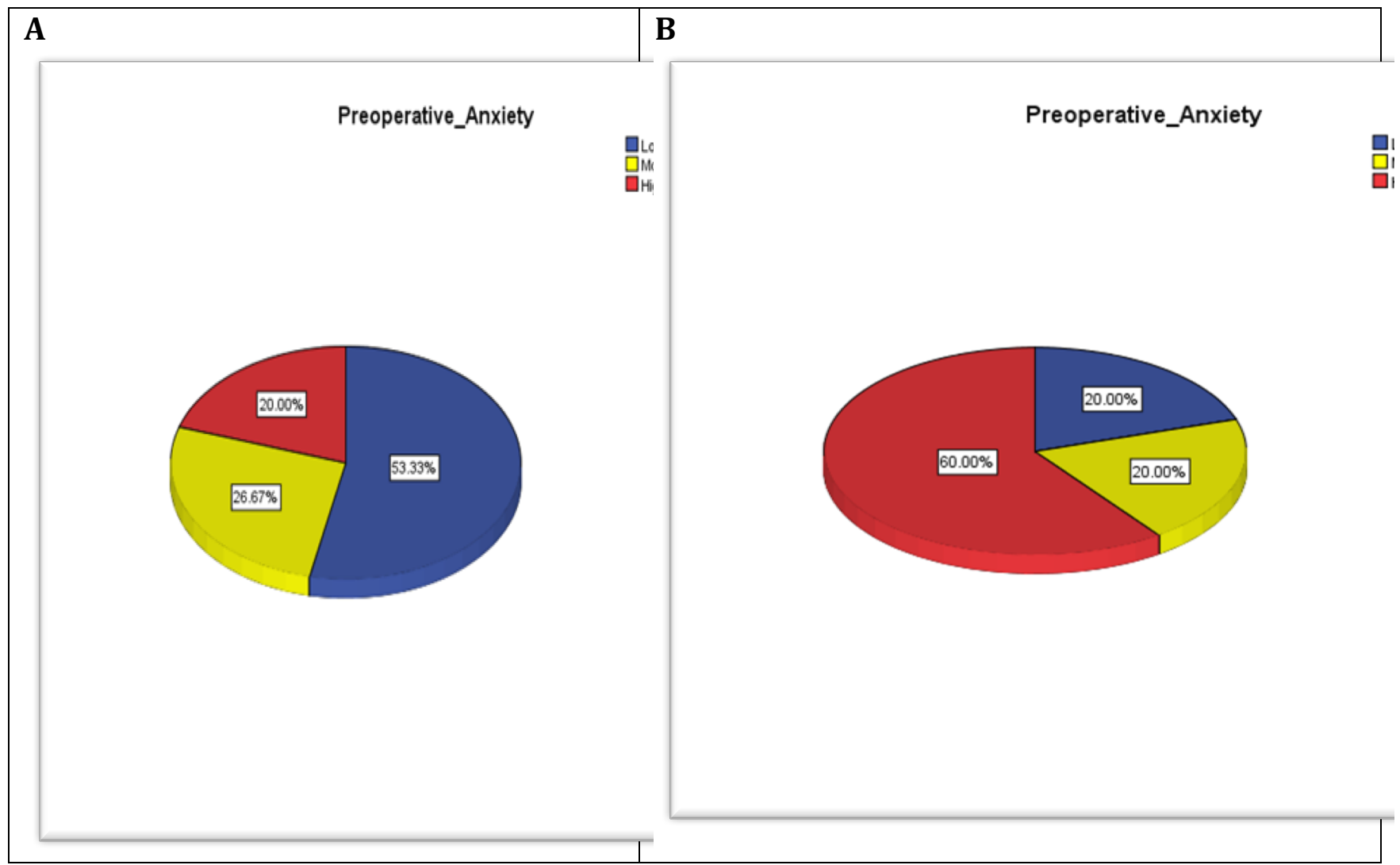

Figure 4: A= Participants' levels of preoperative anxiety measured during the evening before surgery day. $B=$ Participants' levels of preoperative anxiety measured on the morning of their surgery day.

\section{DISCUSSIONS}

The primary aim of this study was to assess the prevalence of preoperative anxiety among Hausa patients before undergoing elective surgery. This section discusses the findings of the study in the context of the researches previously published. The discussion is structured according to the way the data has been analysed where preoperative anxiety has been assessed in accordance with the sub-scales of the APAIS-H. The full scale anxiety scores were also discussed to provide general information on the participants' preoperative anxiety.

The results of this study show that, majority of the participants experience high degree of preoperative anxiety. Their anxiety is related to both anaesthesia and the surgical operation. 
The patients' anxiety keeps increasing in intensity as they approach their time of operation. For example, it was found that in the evening before the surgery, on the anxiety related to anaesthesia sub-scale, 10 patients (33.3\%) presented with moderate anxiety and only 7 $(23.3 \%)$ presented with high anxiety related to anaesthesia. In contrast, on the morning of the operation, the patients scores on the anxiety related to anaesthesia sub-scale shows that, only $6(20 \%)$ patients presented with moderate anxiety while $19(63 \%)$ presented with high anxiety related to anaesthesia. This finding is in line with (Jakobsson et al. 2008) who reported that patients fear general anaesthesia, being under the anaesthetic mask and fear of needles during the induction of anaesthesia are the most reported source of preoperative anxiety among their patients. Similarly, Carr et al. (2006) also reported the general pattern of patients preoperative anxiety in their study, where participants anxiety rose during the preoperative period to reach a peak prior to the anaesthetic, and then decreased one day after the operation.

On the anxiety related to surgery sub-scale, on the evening before the surgery, 10 (33.3\%) patients had low levels of surgical anxiety, while 20 patients $(66.60 \%)$ reported having moderate to high levels of anxiety related to surgery. On the morning of the day of surgery, the Patients' APAIS-H scores significantly increased. Only $4(13.3 \%)$ reported having low anxiety related to surgery; 8 (26.7\%) presented with moderate surgical anxiety and a greater proportion $18(60 \%)$ of the participants recorded having high levels of anxiety related to surgery. This finding is similar to the results reported by Jafar and Khan (2009) who studied the frequency of preoperative anxiety in Pakistani surgical patients. Their study results revealed that $62 \%$ of their total participants were significantly anxious preoperatively.

The participants need for information about surgery also shows a similar pattern with results for anxiety related to anaesthesia and surgery. Participants' scores on the information desire sub-scale revealed that in the evening preceding the day of surgery, only 5 participants $(16.7 \%)$ required low information about their surgery, 8 participants (26.7\%) needed moderate information about the surgery, and a high number of participants (17 - 56.7\%) reported a higher need for information about their surgery. On the other hand, on the morning of the day of the surgery, no patient reported having low desire for surgical information. Patients reported between moderate and higher need for information, with only 7 patients $(23.3 \%)$ presenting with moderate need for information, while the remaining 27 participants (76.7\%) reported higher desire for surgical information. This result is similar to what (Sjöstedt et al. 2011) reported in which they reported four types of information desire among their participants - the need for information to reduce pre and post-operative anxiety, the need for information for a sense of assurance, information regarding the management of postoperative pain and provision of information about general knowledge of surgery. Sjöstedt et al. (2011) concluded that, there is need to supply information to specifically alley patients' anxiety and to create a sense of assurance and understanding of the entire health care procedure.

The present study found high prevalence of preoperative anxiety in the patients studied. The cumulative sum of scales scores of preoperative anxiety, the evening preceding the surgery, shows that, 16 participants (53.3\%) had low level of preoperative anxiety, 8 (26.7\%) had moderate level of anxiety and only $6(20 \%)$ of the participants recorded having high level of preoperative anxiety. On the other hand, on the morning of the surgery day, participants' measured preoperative anxiety reveal equal number of participants (six each) presented with low and moderate levels of preoperative anxiety respectively, while a significant number of participants, $18(60 \%)$ showed higher levels of preoperative anxiety. A similar result was reported by (Gangadharan et al. 2014) who found that a total of $10 \%$ of their participants reported low preoperative anxiety; $30 \%$ reported moderate anxiety; and a significant number, 
$60 \%$ of their patients reported high anxiety prior to their surgical operation. In the same way, Akinsulore et al. (2015) also reported a finding that revealed 51.0\% of their study participants had exhibited significantly high pre-operative anxiety, whereas only $15.7 \%$ presented with significantly high post-operative anxiety. Also, (Jafar and Khan 2009) reported higher rate of preoperative anxiety in $62 \%$ of their participants before they go in for the surgery.

\section{IMPLICATIONS OF THE STUDY FOR CLINICAL PRACTICE}

Given that medical and psychological literature is replete with information on physical and psychological effects of pre-operative anxiety on surgical patients, it is recommended that adequate assessment of pre-operative anxiety for all surgical patients is made before they undergo a surgical operation. This would provide clinicians with adequate information on Hausa-speaking patients in need of pre-operative anxiety reduction interventions as well as those in need of more information about their surgery. A better understanding of the fundamental issues causing patients' pre-operative anxiety may give clinicians the evidence needed to develop more effective therapeutic interventions to alleviate high anxiety before the patients undergo their surgery. Assessment of patients' pre-operative anxiety also gives clinicians the bases to appropriately diagnose, identify treatment-related patient issues and address those using available resources. Establishing the level of pre-operative anxiety will help the clinicians to explain the type of intervention they need to provide to the patients. Ascertaining the prevalence of preoperative anxiety will help the clinicians not to miss patients who fake and present with low or no anxiety while in the real sense they are more anxious than they pretend and therefore in need of more clinical intervention. The insights from assessments will help the clinicians to form collaborative treatment plans with patients and other clinical partners to address the patients' needs. More so, assessing and classifying patients that are anxious from non-anxious ones would give the clinicians, as well as the hospital authorities, the opportunity to adequately and efficiently use their available resources on only those patients who need them. Overall, given the evidence indicating the connection between pre-operative anxiety and post-operative outcome, it is recommended to include this approach in the pre-operative consultation.

\section{LIMITATIONS OF THE STUDY}

Although the study found that there is high prevalence of preoperative anxiety among the participants studied, the study is not without limitations. First the study recruited only 30 participants, Therefore a larger sample size of Hausa-speaking surgical patients needs to be recruited for future study to provide more accurate data representative of the target population. Although the 30 participants used in the current study are sufficient to produce significant results, engaging a large number of participants would increase the quality of the study and confidence in making a generalisation of the findings.

This study is a single centre study. There is therefore a need to carry out a similar or the same study in two or more centres using the same kind of patients, the same study instrument, procedure for data collection and the same method of statistical analysis. This will provide a good grounding for making a generalisation of the findings of the study.

The study also used patients with different types of elective surgery as participants. The difference in types of surgical cases among the study participants may be a factor for the high levels of pre-operative anxiety recorded. Some patients' surgical cases are not complicated while others are more complicated. This could be a factor responsible for the high preoperative anxiety seen in the study participants. There is therefore a need for a similar study to be conducted using participants with a homogeneous type of elective surgical case so that the 
anxiety score recorded in these patients may not be attributable to differences in surgical procedures but the surgical case at hand.

\section{CONCLUSION}

In conclusion, the present study found that, preoperative anxiety is very prevalent in patients waiting to undergo elective surgery and that the patients' anxiety as well as their need for surgical information amplified as they near the operation. The study did not find difference in preoperative anxiety among male and female participants. However, there was a significant difference in terms of desire for information with female participants desiring more information than their male counterparts

\section{References}

Akinsulore, A., Owojuyigbe, A. M., Faponle, A. F. and Fatoye, F. O. (2015) assessment of preoperative and postoperative anxiety among elective major surgery patients in a tertiary hospital in nigeria. Middle East Journal of Anesthesiology 23 (2), 235-240.

Ali, A., Altun, D., Oguz, B. H., Ilhan, M., Demircan, F. and Koltka, K. (2014) The effect of preoperative anxiety on postoperative analgesia and anesthesia recovery in patients undergoing laparascopic cholecystectomy. Journal of anesthesia 28 (2), 222-227.

Aziato, L. and Adejumo, 0. (2014) An Insight Into the Preoperative Experiences of Ghanaian General Surgical Patients. Clinical nursing research 23 (2), 171-187.

Bailey, L. (2010) Strategies for decreasing patient anxiety in the perioperative setting. AORN journal 92 (4), 445460.

Bilberg, R., Nørgaard, B., Overgaard, S. and Roessler, K. K. (2012) Patient anxiety and concern as predictors for the perceived quality of treatment and patient reported outcome (PRO) in orthopaedic surgery. BMC Health Services Research 12, 244.

Carr, E., Brockbank, K., Allen, S. and Strike, P. (2006) Patterns and frequency of anxiety in women undergoing gynaecological surgery. Journal of clinical nursing 15 (3), 341-352.

G. Chakir, A. G. (2017) The Effect of Pre-Operative Distress on the Perioperative Period. Journal of Anaesthesia and Intensive Care Medicine 2 (3), 001-003.

Gangadharan, P., Assiri, R. A. M. and Assiri, F. A. A. (2014) evaluating the level of anxiety among pre-operative patients before elective surgery at selected hospitals in kingdom of saudi arabia. Int J Cur Res Rev/ Vol 6 (22), 37.

Jafar, M. F. and Khan, F. A. (2009) Frequency of preoperative anxiety in Pakistani surgical patients. Journal of the Pakistan Medical Association 59 (6), 359.

Jakobsson, J., Stomberg, M. W., Rawal, N., Brattwall, M. and Segerdahl, M. (2008) Day surgery for knee arthroscopy, open hernia repair and laparoscopic cholecystectomy anaesthesia routine and practice; The results from a Swedish nation-wide survey. Ambulatory Surgery 14 (2), 1-23.

Matthias, A. T. and Samarasekera, D. N. (2012) Preoperative anxiety in surgical patients-experience of a single unit. Acta Anaesthesiologica Taiwanica 50 (1), 3-6.

May, M. (2014) One in 25 Patients End Up with Hospital-Acquired Infections.

http://articles.mercola.com/sites/articles/archive/2014/04/09/hospital-acquired-infections.aspx Accessed 15/04/2017.

Mitchell, M. (2009) Patient anxiety and conscious surgery. Journal of perioperative practice 19 (6), $168-173$.

Moerman, N., van Dam, F. S., Muller, M. J. and Oosting, H. (1996) The Amsterdam preoperative anxiety and information scale (APAIS). Anesthesia \& Analgesia 82 (3), 445-451.

NHS (2015) General anaesthesia. 08/05/2015. NHS. http://www.nhs.uk/conditions/Anaestheticgeneral/Pages/Definition.aspx Accessed 05/06/201.

Perks, A., Chakravarti, S. and Manninen, P. (2009) Preoperative anxiety in neurosurgical patients. Journal of neurosurgical anesthesiology 21 (2), 127-130.

Pritchard, M. J. (2009a) Identifying and assessing anxiety in pre-operative patients. Nursing standard 23 (51), 3540. 
Pritchard, M. J. (2009b) Managing Anxiety in the Elective Surgical Patient. British Journal of Nursing 18 (7), $416-$ 419.

Sjöstedt, L., Hellström, R. and Stomberg, M. W. (2011) Patients' need for information prior to colonic surgery. Gastroenterology nursing 34 (5), 390-397.

van den Berg, A. A., Chitty, D. A., Jones, R. D., Sohel, M. S. and Shahen, A. (2005) Intravenous or inhaled induction of anesthesia in adults? An audit of preoperative patient preferences. Anesthesia \& Analgesia 100 (5), $1422-1424$.

Wakim, J. H., Smith, S. and Guinn, C. (2010) The Efficacy of Music Therapy. Journal of PeriAnesthesia Nursing 25 (4), 226-232.

Ward, C., Varvinski, A. and Montgomery, J. (2007) Patients' Choice of Induction Method: Do Patients Prefer Being Given a Choice of their Induction Method? Journal of One Day Surgery 17 (2), 33.

Wetsch, W. A., Pircher, I., Lederer, W., Kinzl, J., Traweger, C., Heinz-Erian, P. and Benzer, A. (2009) Preoperative stress and anxiety in day-care patients and inpatients undergoing fast-track surgery. British journal of anaesthesia 103 (2), 199-205.

Willson, R. and Veale, D. (2009) Overcoming health anxiety: a self-help guide using cognitive behavioral techniques. London: Robinson. 